\title{
Are the measurements of water-filled and air-charged catheters the same in urodynamics? Response to comments
}

\author{
G. Alessandro Digesu
}

Published online: 10 October 2013

(C) The International Urogynecological Association 2013

\section{Dear Editor,}

We appreciate Dr. Valentini's interest [1] in our article [2]. Her criticisms of our study are essentially that: (1) The use of two catheters to measure simultaneously intravesical pressure leads to nonphysiological conditions. This, in addition to a possible interaction between the two catheters, may affect the pressures measured. (2) Detrusor pressure should not be compared in the study. (3) Vesical pressure measured by the water-filled catheter is higher than the vesical pressure measured by the air-charged catheter. We address each of these three points below.

1. We completely agree with the author, and this was emphasized at the end of our Discussion section. Although we may accept the author's criticism that this should have been discussed in more detail, we would not consider it a significant flaw to our study. We also believe that during routine cystometry, having even one urethral catheter is a nonphysiological condition anyway. Second, if there is an artificial effect caused by inserting two catheters in the bladder to simultaneously measure intravesical pressure, we would expect both intravesical pressures (measured by air-charged and water-filled catheters) to be affected equally. Finally, performing measurements separately may not allow a reliable comparison of pressures, which may have different values if they are measured on different times. The reason the pressures were measured simultaneously in our study, as well as the limitation of such measurement, was also clearly explained in the Discussion section.

2. The difference In detrusor pressures measured by the aircharged and water-filled catheters was included in the manuscript for completeness of the results. As detrusor pressure is a subtracted pressure, it is clear that a different vesical and/or abdominal pressure will affect detrusor pressure reading. The source of discrepancies between readings measured by the two catheters were also clarified and explained in the Discussion.

3. The author appropriately interpreted the trace shown in Fig. 1 of our manuscript. This confirmed that pressures measured by the two catheters were different.

In summary, we do not think that there are any significant flaws in our study, and the issues mentioned by Dr. Valentini do not introduce bias in any direction. The aim of our study was not to determine the best catheter to use during urodynamics. Based on our results, we cannot determine whether the different readings obtained by the two methods were clinically significant. Our aim was only to assess whether they can reproduce comparable pressure readings. As the two catheters measured different pressures, we concluded that urodynamic parameters must not be compared if different catheters are used. The catheters cannot be used interchangeably, and a uniform type of catheter must be used in multicenter studies.

\section{References}

1. Valentini FA (2013) Comments on: Are the measurements of waterfilled and air-charged catheters the same in urodynamics? Int Urogynecol J. doi:10.1007/s00192-013-2236-2

2. Digesu GA, Derpapas A, Robshaw P, Vijaya G, Hendricken C, Khullar V (2013) Are the measurements of water-filled and air-charged catheters the same in urodynamics? Int Urogynecol J. doi:10.1007/s00192$013-2182-z$

G. A. Digesu $(\bowtie)$

Department of Urogynecology, St Mary's Hospital, London, UK

e-mail: a.digesu@imperial.ac.uk 